\title{
The relationship between central cues and peripheral cues in covert visual orientation
}

\author{
LUCIA RIGGIO \\ University of Parma, Parma, Italy \\ and \\ KIM KIRSNER \\ University of Western Australia, Nedlands, Australia
}

\begin{abstract}
Four experiments were conducted to compare valid and invalid cue conditions for peripheral and central cues. Experiments 1, 3, and 4 used reaction time (RT) as the dependent variable. Experiment 2 used a threshold measure. Peripheral and central cues were presented on each trial. The peripheral cue was uninformative in all experiments. The central cue was informative in Experiments 1 and 2, where it predicted stimulus side on $70 \%$ of the trials. Experiment 3 included $50 \%$ and $100 \%$ central-cue prediction conditions as well as the $70 \%$ treatment. Experiment 4 included $60 \%, 75 \%$, and $90 \%$ central-cue prediction conditions. The effects of the central and peripheral cues were independent and additive in all four experiments, indicating that: (1) both cue types can act simultaneously, and that the relationship between them is additive under the conditions used in these experiments, (2) informativeness is not a necessary condition for attentional effects with peripheral cues, and (3) covert visual orientation influences sensory thresholds and RT in similar ways. The results of Experiments 3 and 4 demonstrated that the facilitation associated with peripheral cues was insensitive to manipulations which demonstrate that subjects use the informational value of the central cue to direct voluntary attention. The results are discussed with reference to two issues; first, the proposition that central and peripheral cues exert their influence on performance in independent information-processing stages, following the additive factor method, and, second, the problems raised for additive factors method when cues elicit both an "explicit" response-regarding the presence or absence of a specified letter-and an "implicit response"-involving the planning and possible execution of eye and hand movements.
\end{abstract}

There is extensive evidence that people can shift the focus of their attention to extrafoveal loci without moving their eyes (Eriksen \& Colegate, 1971; Eriksen \& Hoffman, 1972; Posner, Snyder, \& Davidson, 1980). This process has been described as "covert visual orienting" to distinguish it from its obvious partner, overt visual orienting (Posner, 1980). The ecological value of covert visual orienting has not been specified, although cognitive and behavioral economy provide obvious candidates. The behavioral advantage stems from an interpretation in which many objects are given temporary status in, say, visual working memory, but only a small fraction of these objects are followed by either eye or eye and head movements. The cognitive case is the same, except that economic selection applies to further visual or cognitive processing rather than overt behavior.

Covert visual orienting may be captured or controlled in either of two ways, one involving peripheral cues, and

Preliminary results of Experiments 1 and 2 were presented at XII Congresso Nazionale Divisione Ricerca di Base in Psicologia, Rome, September 1993. The authors wish to thank C. Umiltà and B. M. Sheliga for a critical reading of the manuscript and $P$. Standen and G. Ferrari for technical assistance. Correspondence should be directed to L. Riggio, Istituto di Fisiologia Umana, Università di Parma, Via Gramsci 14, 43100 Parma, Italy (e-mail: riggio@ipruniv.cce.unipr.it). the other, central cues. Experiments involving peripheral cues have used, for example, abrupt stimulus onset, abrupt stimulus offset, and other luminance transitions. Typically, these stimulus manipulations are presented at or near the point at which the test stimulus is subsequently presented. In general, the effect of these treatments is to reduce reaction time (RT) on detection, identification, and classification tasks to stimuli that are subsequently presented at these locations as compared with carefully selected control conditions. Two types of control conditions have usually been used. The first, or neutral, control condition involves the absence of a spatially selective cue so that predictive information about the position of the test stimulus is not available at all. The second, or invalid, control condition involves presentation of a cue that provides predictive information about the position of the subsequent test stimulus which proves to be incorrect because the test stimulus is presented in some other position. The first of the above conditions, when the cue has correct predictive value, is called the valid condition.

Experiments involving central cues involve the same manipulations as those described above except that the spatial cue is typically provided symbolically and in the center of the visual field. Thus, a left-pointing arrow (i.e., $\leftarrow$ ) presented in the center of the visual field is logically equivalent to a luminance transient at some arbitrary but con- 
sistent position in the left hemifield. In the case of the central cue, of course, the cue must be "read" and interpreted before covert visual orientation can occur, but the two modes of directing attention are logically similar; the subject is provided with a cue that, with some probability value, provides predictive information about the spatial location of a subsequent test stimulus. The central-cue procedure also typically uses the valid, neutral, and invalid conditions described above.

The performance characteristics associated with central and peripheral cues are not identical. Valid peripheral cues drive rapid and automatic facilitation (e.g., Maylor, 1985; Posner \& Cohen, 1984; Possamaï, 1986), whereas that associated with a valid central cue is slower and subject to voluntary control (e.g., Jonides, 1981; Müller \& Findlay, 1988; Müller \& Rabbitt, 1989). There is evidence, furthermore, that the facilitation associated with a peripheral cue is followed by inhibition when the cue is not informative of the spatial location of the stimulus (e.g., Maylor, 1985; Maylor \& Hockey, 1985; Posner \& Cohen, 1984; Possamaï, 1986; Tassinari, Aglioti, Chelazzi, Marzi, \& Berlucchi, 1987). These features are not observed when central cues are used.

An important question concerns the extent to which central and peripheral cues involve reference to the same information-processing stages and processes. Consider, for example, the study by Briand and Klein (1987). Subjects were cued to pay attention to a particular location by visual cues (central or peripheral in different experiments), and they had to decide if an $|R|$ was present or not in a pair of letters. Two different sets of letters were used. In the "feature" set, the distractors were the letters P and $\mathrm{B}$, so that only the letter target had the discriminating feature of the oblique line. In the "conjunction" set, the distractors were the letters $P$ and $Q$, so that the obliqueline feature was shared also by the letter $Q$. The two sets were included in order to exploit conditions that favored and precluded the use of focused attention. The feature set supported detection, without the intervention of focused attention, by an early, automatic, and parallel registration of the features. The conjunction set consisted of letters that did not support detection without the intervention of focused attention. $|R|$ and $|Q|$ can be identified unambiguously only under focused-attention conditions. It is then that critical features can be integrated (i.e., in a "percept") and illusory conjunctions of features avoided (e.g., Treisman \& Gelade, 1980; Treisman \& Schmidt, 1982). Results showed similar effects for both sets when attention was oriented with central cues, but when peripheral cues were used, conjunction set showed larger effect of cuing than did feature set (see also Prinzmetal, Presti, \& Posner, 1986; Treisman, 1985). The conclusions were that peripheral and central cues could orient different attentional systems, and that only the system oriented by peripheral cues performed a role in featurebased integration or inhibition (Treisman, 1985; Treisman \& Sato, 1990).

Müller and his colleagues (Müller \& Findlay, 1987; Müller \& Humphreys, 1991; Müller \& Rabbitt, 1989) have proposed the existence of two functionally independent (reflexive and voluntary) orienting mechanisms addressing the same limited-capacity attentional system. Peripheral cues first engage the fast reflexive mechanisms and then, if informative, the slower voluntary mechanisms. Central cues are related only to the voluntary component. The two orienting mechanisms interact in a mutually inhibitory way (Müller \& Humphreys, 1991). The model, therefore, correlates peripheral and central cues with orienting of attention and not with the processes of attention.

Studies directly contrasting voluntary and automatic control of attention have shown mixed results (e.g., Koshino, Warner, \& Juola, 1992; Müller \& Rabbitt, 1989; Remington, Johnston, \& Yantis, 1992; Theeuwes, 1991; Warner, Juola, \& Koshino, 1990; Yantis \& Jonides, 1990). Even if there is general agreement that peripheral cues have an automatic component, the degree of automaticity and, therefore of the separability of central and peripheral cuing processes, has been debated. According to some researchers (Theeuwes, 1991; Warner et al., 1990; Yantis \& Jonides, 1990), the establishment of a particular expectancy can override the effects of peripheral cues. On the other hand, Remington et al. (1992) showed that when subjects had to ignore irrelevant abrupt-onset stimuli whose spatial relation to the target they knew, they could not do it. In this case, the establishment of a negative set for a stimulus could not override the capture of attention by the stimulus itself.

The method described by Sternberg (1969) provides a framework for the investigation of hypotheses about the relationships among the stages engaged by central and peripheral cues. Sternberg described a method for identifying the stages involved in performance tasks and a retrieval model involving four stages for a particular task involving short-term recognition memory. The model was predicated on the assumption that information processing involved a series of distinct mental processes, each of which was responsible for a different function. According to Sternberg's application of the additive factors method to short-term recognition memory, or "character classification," the stages involved in this task were encoding the test stimulus, memory search, decision (old or new), and response selection and execution. The model was based on evidence that variables associated with each of these stages had additive effects on the data, although the method of selective influence introduced by Sternberg could support inferences only about stage existence and not stage order.

Figure 1 includes a depiction of Sternberg's model. Following Sanders (1990), the central column in Figure 1 depicts seven stages. The first three stages involve perceptual processes, the fifth stage involves a decision process concerning response selection, and the final two stages involve response processes. Following Sternberg, and because the letter classification task used in our experiments involves a mapping function (i.e., to assign each stimulus with a specific decision category), we have also included binary decision as a distinct stage. The model 


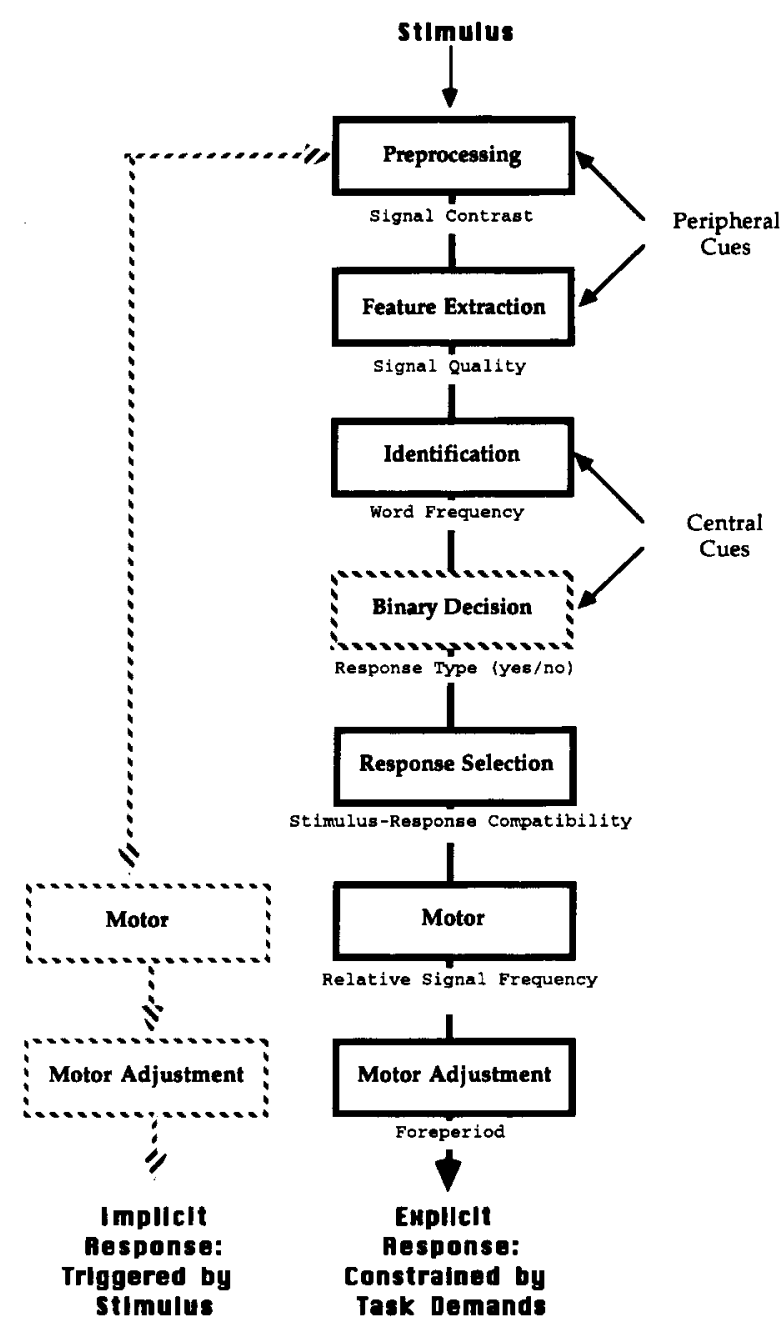

Figure 1. Depiction of Sternberg's (1969) stage model based on Sanders (1990). The central sequence involves stages in the explicit route. The left-hand sequence involves hypothetical stages for ocular and manual responses associated with the implicit route. The dashed boxes are additions to those described by Sanders. Labels in small print identify variables associated with each stage.

also includes one variable for each stage, providing an illustration of the type of manipulation that influences performance in each stage. These variables were selected for illustrative purposes from the larger sets identified by Sternberg (1969) and Sanders (1990) for each stage. Unlike Sanders's account, we have ignored cases where a given variable interacts with variables associated with more than one stage.

Figure 1 includes a provisional analysis of the possible locus of central- and peripheral-cue effects. As depicted in that figure, peripheral cues trigger a change in attention where the product of that change influences efficiency during either preprocessing or, possibly, the later feature-analysis stage, the position advanced by Klein and his associates (e.g., Briand \& Klein, 1987; Klein, Kingstone, \& Pontefract, 1992). It should be noted that we are not concerned with analysis of the peripheral cue as such, but with the influence of that analysis on letter recognition.

Sanders (1990) does not associate a specific stage with such voluntary control processes as expectancy, and the central-cue manipulation does not therefore have an obvious parallel with any of the variables described by him. Central cues clearly depend on voluntary or top-down processes, however, and it is therefore appropriate to associate them with such decision processes as binary decision and response selection, although a late perceptual process such as identification cannot be excluded.

Two types of prediction merit consideration. The first of these involves the relationship between peripheral and central cues. If the products of the processes provoked by these cues influence the same stage, it may be predicted that these variables will interact. However, if central and peripheral cue variables influence separate stages, it may be predicted that the relationship between these variables will be additive. The second of these hypotheses is illustrated in Figure 1, where the peripheral- and central-cue manipulations are assigned to specific pairs of stages. One advantage of this approach is that it provides for the possibility of interactions involving such other variables as letter set and response type (yes or no), a step that could be used to associate the variables of interest with other variables and, therefore, other stages.

In summary, the critical issue emphasized by Figure 1 concerns the presence and locus of convergence between the influence of the central and peripheral cues. Figure 1 depicts a situation in which peripheral and central cues each provoke changes in information-processing efficiency. It is further suggested that the efficiency changes provoked by these cues occur at different stages, and that following the additive factor method - their contributions will be additive and independent. We have provisionally assigned the influence of peripheral cuing to the preprocessing, or feature-extraction, stages and central cuing to the identification, or response-selection, stages as specified in Sanders's (1990) model. It should be noted that this assignment is not required by our central hypothesis. The critical test concerns the hypothesis that peripheral and central cues influence different information-processing stages.

Given the foregoing analysis, it is possible to devise a formal test of the relationship between central and peripheral cues using a variation of Sternberg's additive factor method. If our hypothesis is correct, the relationship between the peripheral- and central-cue manipulations should be additive.

\section{EXPERIMENT 1}

Experiment 1 was designed to compare valid and invalid cue conditions for peripheral and central cues. Each trial included both peripheral and central cues. The valid and invalid conditions were combined orthogonally. The central cues were informative; that is, they provided information that could be used to predict the position of the imperative stimuli. The peripheral cues were uninforma- 
tive: they did not provide spatial information about the imperative stimulus. An RT task was used. The intervals between the cues and the imperative stimuli were selected so as to ensure that each variable influenced performance, and to establish conditions where these variables would have more or less equal weight, a priori.

A modification of Briand and Klein's (1987) paradigm was adopted. A letter-detection task was used. Two letters were presented on each trial. The letters were shown side by side in the left $o r$ the right visual field, and subjects were required to indicate whether or not the letter $|R|$ was present. Each trial also included presentation of a valid or invalid central cue and a valid or invalid peripheral cue, where the cue treatments were chosen to maximize the likelihood of obtaining effects of each type. Thus, the central cue was presented $1,140 \mathrm{msec}$ in advance of the test stimulus, in order to provide adequate time for the development and establishment of voluntary orientation. The peripheral cue was presented only $140 \mathrm{msec}$ in advance of the test stimuli, at an interval sufficient for reflexive orientation.

Two stimulus sets were used, with the letters RPB and the letters RPQ, respectively (see above). If only the attentional mechanisms oriented by peripheral cues have a role in integrating features in objects, we could expect different validity effects for the two letter sets when testing performance related to peripheral cues.

\section{Method}

Subjects. Twelve right-handed (Oldfield, 1971) female students, all 18 years old, from the University of Western Australia acted as subjects. All of the subjects had normal or corrected vision, and all were ignorant as to the purpose of the experiment. The subjects were paid for their services.

Apparatus. The experiment was controlled by a PDP-11/60 computer. The computer managed stimulus display, timing, data collection, and data storage. Responses were made by pressing a key on a two-key response-board; the two keys were in symmetrical positions, each of them about $20 \mathrm{~cm}$ from the body midline, and operated by the index fingers of the subjects' hands held in anatomical positions. The subjects were requested to keep their index fingers on the keys all the time. During the experiment, the subjects' heads rested on an adjustable chinrest to maintain the distance between their eyes and the screen at about $50 \mathrm{~cm}$.

The stimuli were presented on a Tektronix 620 oscilloscope with P31 phosphor. Stimuli were the capital letters R, P, Q, and B; they were formed by $67,57,77$, and 77 points, respectively, and their outlines were inscribed in a $54 \times 80$ point matrix $\left(0.62^{\circ} \times 0.92^{\circ}\right.$ visual angle).

Design. The experiment consisted of 640 trials, divided into 16 blocks of 40 , with some minutes' rest in between, run in one session. Table 1 is a summary of the design showing the number of trials under each combination of cue type (central or peripheral), cue direction (left or right), and cue validity (valid or invalid) for each letter set.

The central cues were followed by valid and invalid test stimuli on $70 \%$ and $30 \%$ of the trials, respectively. The peripheral cue was uninformative; it was followed randomly by valid and invalid trials.

Procedure. Each letter set was tested in eight successive blocks of 40 trials preceded by a block of practice trials. Six subjects had the RPB letter set in the first eight blocks and the RPQ letter set in the last eight blocks. The reverse was true for the remaining 6 subjects.

Each trial started when three dots appeared on the screen. The dots were aligned horizontally. The central dot corresponded to the geo-
Table 1

Summary of Design for Each Letter Set Specifying the

Directional Value of Each Cue (Left or Right) and the Frequency of Each Type of Trial

\begin{tabular}{lllcc}
\hline \multicolumn{2}{c}{ Type of Cue } & & \multicolumn{2}{c}{ Position of Stimulus } \\
\cline { 5 - 5 } Central & Peripheral & & Left & Right \\
\hline Left & Left & 56 & 24 \\
Left & Right & 56 & 24 \\
Right & Left & 24 & 56 \\
Right & Right & 24 & 56 \\
Total & & 160 & 160 \\
\hline
\end{tabular}

metric center of the screen; the other two dots were presented $2.86^{\circ}$ of visual angle to the left and right of the fixation position.

The central cues consisted of an arrow pointing left or an arrow pointing right, and the peripheral cues consisted of the brightening and expansion of one of the peripheral dots. The subjects were instructed to pay attention to the side indicated by the arrow and to ignore the peripheral cues without moving their eyes from the fixation position. Peripheral and central cues were varied orthogonally, so that each condition of a cue could be matched to any condition of the other cue.

Eye movements were monitored by a closed-circuit magnifying TV camera, situated above the screen, and directed on the subject's right eye. When an eye movement was detected during the trial, that trial was discarded and not replaced. Eye movements were rare (less than $1 \%$ ), and they were not analyzed.

An example of the stimulus sequence is depicted in Figure 2. The subjects were instructed to fixate the central dot. After $500 \mathrm{msec}$, the central cue $\left(60 \times 40\right.$ points, $0.69^{\circ} \times 0.46^{\circ}$ visual angle $)$ was superimposed on the fixation dot, and it remained on the screen until the end of the trial. One thousand milliseconds after presentation of the central cue, one of the peripheral dots brightened and expanded to an area of $30 \times 30$ points $\left(0.35^{\circ} \times 0.35^{\circ}\right.$ visual angle $)$ for $40 \mathrm{msec}$ while the central cue remained in view. Following a further period of $100 \mathrm{msec}$, two different letters chosen from the set appeared for $100 \mathrm{msec}$ in the position occupied by either the left or the right dot in such a way that the dot could be thought of as the center of the imaginary rectangle in which the letters were inscribed. Each pair of letters was separated by a gap of 26 points $\left(0.30^{\circ}\right.$ visual angle). At the corresponding area in the alternative hemifield, a series of 50 random points were shown at the same time and for the same period as the stimulus letters. The display remained on until a response occurred, or for $1,200 \mathrm{msec}$. The intertrial interval, measured from onset of the subject's response to the appearance of the three dots for the next trial, was $1,500 \mathrm{msec}$

The subjects' task was to decide whether or not the letter $\mathrm{R}$ was presented on each trial. The letter $\mathrm{R}$ was actually presented on $50 \%$ of trials. Half of the subjects had to press the key on the right if an $R$ was detected and the key on the left if an $R$ was not detected. The reverse was true for the remaining subjects. Speed and accuracy were given equal emphasis.

RTs shorter than $150 \mathrm{msec}$ or longer than $1,200 \mathrm{msec}$ were considered errors (anticipations and retardations) and were discarded.

\section{Results}

The correct mean response latencies and accuracies (following arcsine transformations) were entered into two analyses of variance (ANOVAs) with the following within-subjects factors: letter set (RPB and RPQ), central cue (valid and invalid conditions), peripheral cue (valid and invalid conditions), hemifield (left and right), and response type (yes and no). 


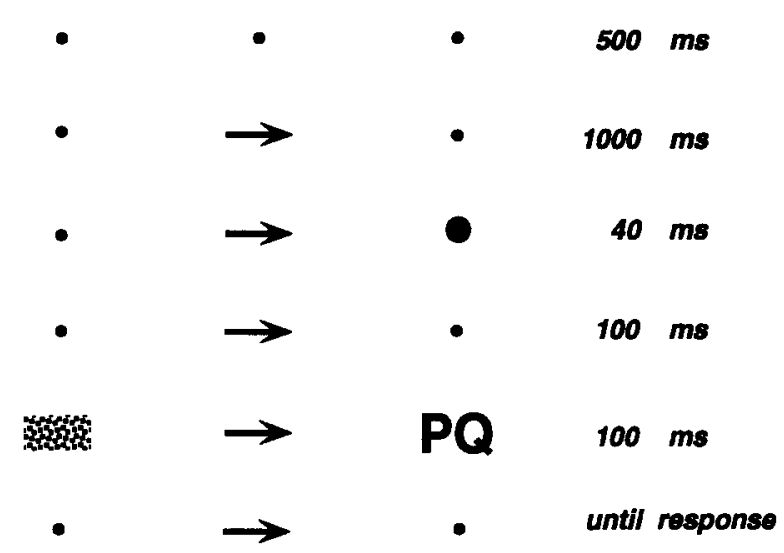

Figure 2. Event sequence and example of display for Experiment 1.

An alpha level of .01 was used for all analyses except for the critical interactions, where additivity was under review, and the exact probability values are shown. Whenever appropriate, post hoc analyses were conducted with the Newman-Keuls method.

Reaction time. The results for central- and peripheralcue validity variables are summarized in Figure 3 . The figure depicts effects for both the central- and peripheralcue types, and shows that the effects of these variables were independent. The ANOVA indicated that the main effects of each cue type were significant $[F(1,11)=13.43$, $p<.01$, for the central cue, and $F(1,11)=37.05, p<.01$, for the peripheral cue] and that the interaction between cue types was not significant $[F(1,11)=0.09, p>.25]$. The differences between the valid and invalid conditions were $25 \mathrm{msec}$ ( $585 \mathrm{vs} .610 \mathrm{msec}$ ) and $21 \mathrm{msec}$ ( $587 \mathrm{vs.}$ $608 \mathrm{msec}$ ) for the central and peripheral cues, respectively.

The effect of hemifield was significant $[F(1,11)=$ $16.65, p<.01]$, with the right hemifield $15 \mathrm{msec}$ faster than the left hemifield ( $590 \mathrm{vs} .605 \mathrm{msec}$ ). The interaction between hemifield and central cue was also significant $[F(1,11)=9.93, p<.01]$. RT for the right hemifield was shorter than RT for the left hemifield under both valid (581 vs. $589 \mathrm{msec} ; .01<p<.05$ ) and invalid conditions (599 vs. $621 \mathrm{msec} ; p<.01$ ). While the general disadvantage experienced by presentation of a letter to the left visual hemifield could be expected on the basis of widely accepted views about hemisphere specialization, the fact that this variable interacts with an attentional variable involving the central cue and showing larger cuing effects for the left than for the right hemifield suggests, instead, that the hemifield differences flow from asymmetrical biases in attention rather than hemifield differences per se (Castiello \& Umiltà, 1992; Gawryszewski, Riggio, Rizzolatti, \& Umiltà, 1987; Umiltà \& Nicoletti, 1985).

The effect of response type was also significant $[F(1,11)$ $=11.27, p<.01]$, with yes responses $39 \mathrm{msec}$ faster than the no responses ( $578 \mathrm{vs.} 617 \mathrm{msec}$ ). The interaction between letter set and response type was significant $[F(1,11)$ $=10.70, p<.01]$. Post hoc analyses with the NewmanKeuls method showed that the difference between the two letter sets was significant for the no responses, at 602 and $632 \mathrm{msec}$ for the RPB and RPQ sets, respectively $(p<.01)$. The difference was actually reversed and not significant for yes responses, at 583 and $573 \mathrm{msec}$ for the RPB and RPQ letter sets, respectively. This pattern is consistent with the position outlined in the introduction, that illusory conjunctions are restricted to no decisions involving the RPQ set.

The letter-set variable did not interact with the centraland peripheral-cue variables. Even if the RPQ set supported the possibility of having illusory conjunctions (see above and the main effect of letter set in the accuracy data), our data did not show a different trend of validity for the two letter sets. In particular the interactions between letter set and peripheral cue, between letter set and central cue, and between letter set, central cue, and peripheral cue were not significant ( $p>.25$ in all cases). Table 2 shows the data related to these variables. Given that illusory conjunctions might be expected to selectively affect trials when the target is absent, a separate analysis was conducted on the relevant data (letter set, central cue, peripheral cue, and hemifield). In this analysis, only the main effects of central cue and peripheral cue were significant.

Accuracy. The main effect of letter set was significant $[F(1,11)=14.67, p<.01]$. The values for the RPB and RPQ letter sets were $96.0 \%$ and $93.4 \%$ correct, respectively. It may be noted that this effect was not significant in the RT analysis, RT for the RPB and RPQ letter sets being 593 and $602 \mathrm{msec}$, respectively. The letter-set variable did not interact with central- and peripheral-cue variables ( $p>.25$ in all cases).

The main effect of response type was also significant $[F(1,11)=7.28, p<.01]$. The accuracy values for the yes and no responses were $93.2 \%$ and $96.2 \%$ correct, respectively.

\section{Discussion}

The results indicate that both cues were influencing performance. RT is shorter in the valid condition than in the invalid condition, regardless of cue type. As predicted, the relationship between the central and peripheral cues is additive, and there were no significant interactions involving both central and peripheral cue types. Although the relationship between central cue and hemifield was significant - suggesting that hemifield biases may be a product of controlled processes - neither the two-way interaction between peripheral cue and hemifield nor the three-way interaction involving central cue, peripheral cue, and hemifield were significant. In summary, the results are consistent with the proposition that central and peripheral cues influence distinct information-processing stages.

\section{EXPERIMENT 2}

The results reported above are consistent with the hypothesis that central and peripheral cues influence distinct information-processing stages. As the hypothesis advanced in the introduction assigns the influence of both cue types to perceptual processes, Experiment 2 was de- 


$$
\text { Reaction Time (ms) }
$$

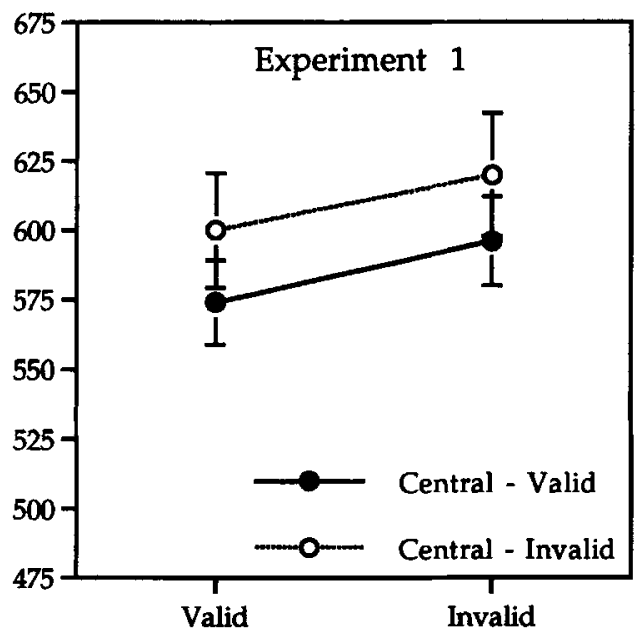

Peripheral Cue
Threshold (ms)

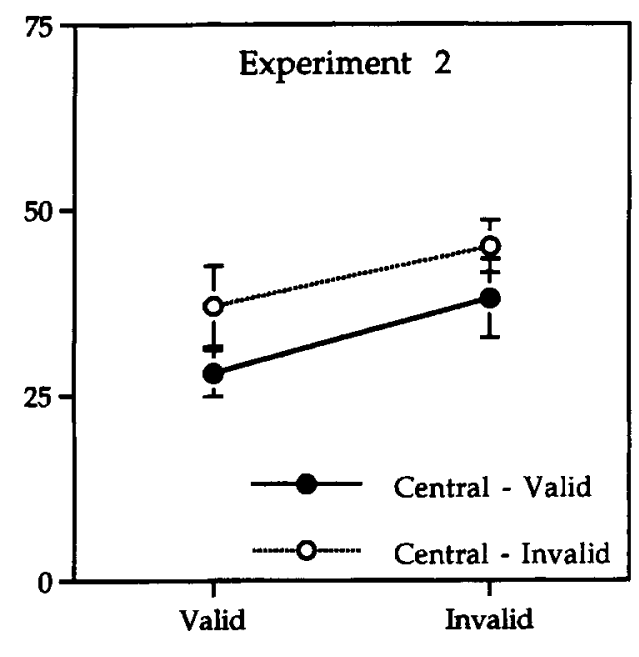

Peripheral Cue
Reaction Time (ms)

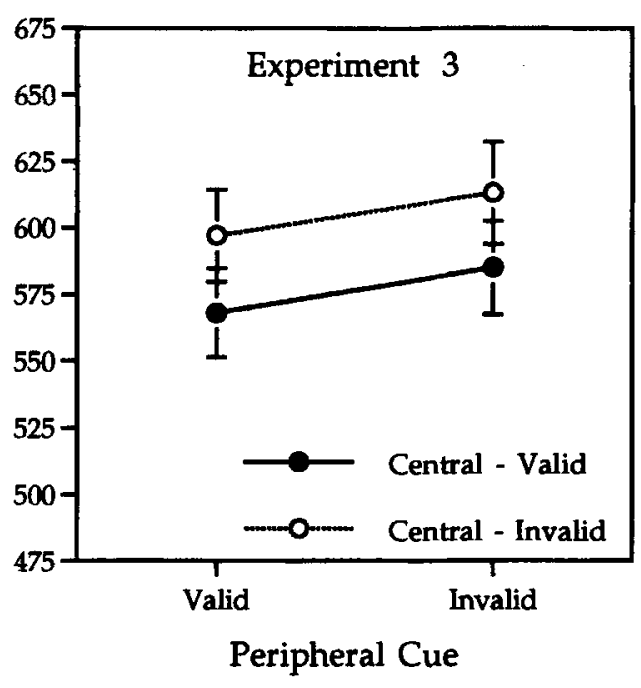

Reaction Time (ms)

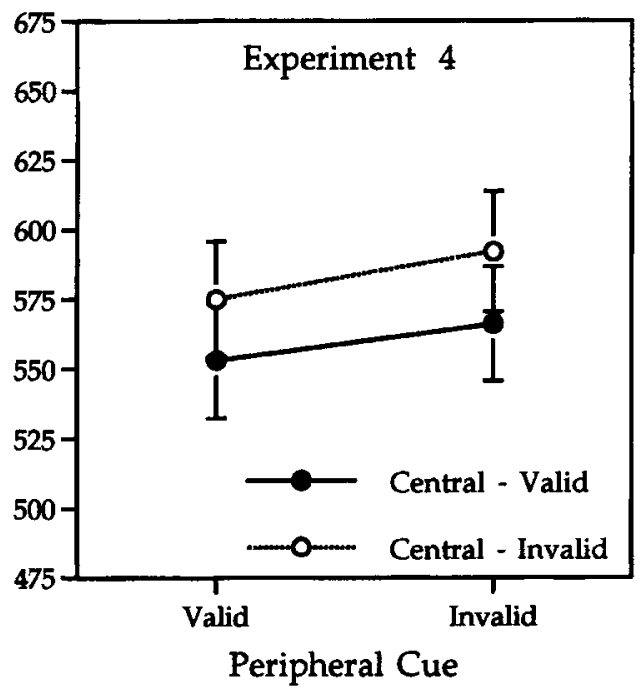

Figure 3. Central- and peripheral-cue effects for Experiment 1 (reaction time), Experiment 2 (threshold), Experiment 3 (reaction time for $70 \%$ central-cue probability), and Experiment 4 (reaction time for all central-cue probability treatments). Bars represent standard errors.

signed to replicate Experiment 1 under conditions that placed more exclusive emphasis on stimulus analysis processes. This was achieved by using a threshold procedure instead of an RT procedure. The logic is, in other respects, similar, although use of a procedure that provides subjects with unlimited response time qualifies application of the additive factor method (see Sanders, 1990). It should be noted that the threshold values involve time, and that the metric is therefore acceptable in this regard. Use of the threshold procedure implicates stimulus analysis rather than production stages such as motor programming, program loading, and motor adjustment (see Figure 1), because a speeded response is not required. The study is mute where the distinction between sensitivity and criterion changes are concerned (see, e.g., Downing, 1988; Hawkins et al., 1990; Müller \& Findlay, 1987).

Experiment 2 was identical to Experiment 1 except that a threshold technique was used instead of the RT technique. A hill-climbing procedure was used to determine separate thresholds for each condition. The threshold procedure involved an adaptation of the parameter estimation by sequential testing procedure (Lieberman \& Pentland, 1982) developed by Standen (1990). The particular adaptation involved a staircase procedure which searches for the stimulus duration at which subjects are able to correctly report stimulus identity at some probability level 
Table 2

Mean Reaction Times (RT, in Milliseconds) and Correct Percentages Under Each Combination of Letter Set, Central Cue, and Peripheral Cue in Experiment 1

\begin{tabular}{ccccccc}
\hline & & \multicolumn{4}{c}{ Peripheral Cue } \\
\cline { 3 - 4 } \cline { 5 - 7 } Letter Set & Central Cue & RT & \% Corr. & & RT & \% Corr. \\
\cline { 3 - 4 } \cline { 6 - 7 } RPB & Valid & 567 & 96.3 & & 592 & 95.9 \\
& Invalid & 597 & 95.5 & & 615 & 96.2 \\
RPQ & Valid & 581 & 94.6 & & 600 & 94.1 \\
& Invalid & 604 & 93.2 & & 625 & 91.7 \\
\hline
\end{tabular}

for each treatment. The threshold study was included to exclude the proposition that peripheral- and central-cue effects depend on the task demands associated with speeded response conditions. If qualitatively comparable cuing results are obtained under threshold and RT conditions, models based purely on RT data will need to be modified accordingly. However, models that specifically include provision for threshold changes as well as premotor planning will not require modification to handle the co-occurrence of threshold and RT effects.

It may be noted that this measure is a sensitivity measure, estimating the amount of time required to solve a perceptual problem, while minimizing the involvement of the information-processing stages associated with production. In this sense, we tried to isolate the components of performance that depend on the sensory processes.

\section{Method}

Subjects. Sixteen right-handed female students (18-22 years old) from the University of Western Australia acted as subjects. All of the subjects had normal or corrected vision, and all were ignorant as to the purpose of the experiment. The subjects were paid for their services.

Apparatus and Procedure. The apparatus, stimuli, and procedure were similar to those used for Experiment 1. The experiment consisted of 640 trials, divided into 16 blocks of 40 , run in only one session. Two groups of 8 subjects were given the RPB and RPQ letter sets.

There were two important differences between Experiments 1 and 2. The first of these differences involved stimulus duration. While the test stimuli in Experiment 1 were always presented for $100 \mathrm{msec}$, stimulus duration in Experiment 2 was controlled by an algorithm designed to determine threshold duration. Based on pilot data, this program commenced with floor and ceiling values of 10 and $60 \mathrm{msec}$, respectively. As chance for the experimental task was $50 \%$ correct, the program searched for the $75 \%$ correct threshold. In general terms, when accuracy fell below $75 \%$ correct for a particular treatment, stimulus duration was increased, but when accuracy was above $75 \%$ correct, stimulus duration was decreased. The program made use of the stimulus (milliseconds) and response (correct or incorrect) values for each trial. The software and display system supported 1-msec steps for stimulus control.

The second difference involved presentation of a poststimulus mask. The mask consisted of letter fragments and occupied the same region as the stimulus and its dummy in the other visual field. The mask was presented for $50 \mathrm{msec}$ immediately following offset of the test stimulus. The procedure therefore determined the $75 \%$ threshold for stimulus onset asynchrony (SOA) for the test stimuli and the mask. Threshold values were determined separately for each experimental treatment and each subject. The response rule followed the earlier experiments; the subjects were instructed to indicate whether or not the letter $R$ was present. The subjects were informed that accuracy was the only measure under consideration, and that they should emphasize this aspect of their performance. Immediate feedback was given to all subjects. Five hundred milliseconds after each response, each subject was presented with a "+" or "-" sign in the middle of the screen, "+" for correct and " -" for incorrect.

The algorithm was searching "simultaneously" for the eight thresholds given by the factorial combination of central-cue validity (valid and invalid conditions), peripheral-cue validity (valid and invalid conditions), and field of presentation (left and right). Data from the yes and no trials for each treatment were required by the algorithm for each treatment (so that each threshold reflected both errors of omission and commission), and this experiment does not therefore provide separate values for yes and no trials.

\section{Results}

The final threshold values (milliseconds) were entered into an ANOVA with the following factors: letter set (RPB and RPQ), central cue, peripheral cue, and hemifield. Except for letter set, all of the factors involved repeated measures. As the threshold values involve an interval scale (i.e., milliseconds), Sternberg's (1969) additive factor logic may be applied to the data, as it is to RT.

The results for central- and peripheral-cue validity variables are summarized in Figure 3. It is apparent that the results replicate the first experiment. There are clear validity effects for each type of cue and little suggestion of any interaction between the central and peripheral conditions. If these values seem small in comparison with RT effects, it should be noted that they involve an improvement of approximately $20 \%$ relative to their control treatments, a value that may be compared with those observed in the RT experiment (i.e., $5 \%$ or less, where RT is approximately $600 \mathrm{msec}$ ).

The interaction between the central- and peripheral-cue conditions was not significant $[F(1,14)=0.12, p>.25]$, an outcome that is consistent with the hypothesis that central and peripheral cues have additive effects. The main effect of validity was significant for the peripheral cue treatment $[F(1,14)=11.57, p<.01]$. However, although the effect of central cue was qualitatively similar to that observed in Experiment 1, the effect was not significant $[F(1,14)=8.74, .01<p<.025]$. The absolute values were 33 and $41 \mathrm{msec}$ for the valid and invalid conditions, respectively, under central-cue conditions and 32 and $42 \mathrm{msec}$ for valid and invalid conditions, respectively, under peripheral-cue conditions.

As in the previous experiment, the main effect of letter set, the interactions between letter set and central cue and between letter set, central cue, and peripheral cue were not significant ( $p>.10$ in all cases). Table 3 shows the data under each combination of letter-set, central-cue, and peripheral-cue variables. There was a trend toward significance for the interaction between letter set and peripheral cue $[F(1,14)=3.71, .01<p<.10]$. As stated above, according to Briand and Klein (1987), only the attentional system oriented by peripheral cues performs a role in feature integration. If this is true, we have to expect the validity effects to be greater in the RPQ set than in the 
Table 3

Threshold Values Under Each Combination of Letter Set, Central Cue, and Peripheral Cue in Experiment 2

\begin{tabular}{cccc}
\hline & & \multicolumn{2}{c}{ Peripheral Cue } \\
\cline { 3 - 4 } Letter Set & Central Cue & Valid & Invalid \\
\hline RPB & Valid & 24 & 35 \\
& Invalid & 26 & 44 \\
\multirow{2}{*}{ RPQ } & Valid & 32 & 42 \\
& Invalid & 47 & 46 \\
\hline
\end{tabular}

RPB set. Actually, we obtained a trend for the opposite result, the difference between valid and invalid conditions ( $40 \mathrm{vs} .44 \mathrm{msec}$ in the valid and invalid conditions), being $4 \mathrm{msec}$ in the RPQ set and $14 \mathrm{msec}$ in the RPB set ( $25 \mathrm{vs}$. $39 \mathrm{msec}$ in the valid and invalid conditions).

We do not have a satisfactory explanation for this difference between our results and Briand and Klein's. Although it is possible that the feature analysis is affected by attentional mechanisms, as claimed by Prinzmetal et al. (1986), letters could act as units, in which case a different explanation would be required for differences between the conjunctive and feature sets (see, e.g., Humphreys \& Bruce, 1989).

\section{EXPERIMENT 3}

Experiments 1 and 2 showed that central and peripheral cues could exert an influence during the same series of experimental trials, and therefore that the distribution of attention in space is the result of the action of both types of cue.

Many recent papers have emphasized the complex relationship between voluntary and automatic control of attentional processes (e.g., Koshino et al.,1992; Müller \& Rabbitt, 1989; Remington et al., 1992; Theeuwes, 1991; Warner et al., 1990; Yantis \& Jonides, 1990). When a subject's attention is in a diffuse or unfocused state, perhaps because no location or object has been selected for attention, peripheral cues always capture attention. When attention is concentrated or focused on a particular object or position by central cues that are valid $100 \%$ of the time - that is to say, when there is certainty on the locus of the stimulus occurrence-peripheral cues have no effect on RT if they are presented outside the focus of attention (Theeuwes, 1991; Yantis \& Jonides, 1990). Yantis and Jonides found that the peripheral stimuli had little or no effect on performance when they were presented inside the focus of attention. However, Theeuwes (1991) found that onset transients disrupted performance if they were presented at the attended location. It appears, therefore, that at present it is still unclear what conditions result in the disruption of performance by peripheral onsets during focused attention.

Nevertheless, the results above seem more in agreement with an interactive than with an additive model of voluntary and automatic attention, and therefore need careful consideration. In Experiments 1 and 2, central cues were never fully informative. It has been suggested that performance depends on cue validity (e.g., Eriksen $\&$ Yeh, 1985; Jonides, 1980; Madden, 1992). The basic idea is that attention involves two states where (1) attention is focused on the cued location, and (2) attention is distributed among the candidate locations. When the validity of the cue is less than $100 \%$, that is to say, when the cue is not fully informative of the location of the target, subjects could use focused attention in a percentage of trials roughly approximate to the validity of the cue (in our case, the validity of the central cue); on the remaining trials, subjects could distribute attention among the candidate locations (Eriksen \& Yeh, 1985; Madden, 1992). If, for example, subjects voluntarily focused attention on the cued location following a central cue, central and peripheral cues could act together (or interact) if they were both valid. But when attention is unfocused, peripheral cues always exert an influence on performance. Thus, the pattern of results found in the first two experiments could reflect the extent to which attention is either focused or unfocused. It is possible, therefore, that peripheral cue effects are sensitive to the proportion of trials in which attention is focused.

In Experiment 3, we introduced two new central-cue conditions, the $100 \%$ and $50 \%$ conditions. In the $100 \%$ probability of central-cue condition, the trials were all valid for the central cue and subjects were therefore motivated to focus attention on the cued location. Under these conditions, then, voluntary attention would be routinely captured by the central cue. In the $70 \%$ probability of central-cue condition, however, the incentive to focus attention in the cued direction is weaker, and the proportion of trials in which attention is focused in that direction should be reduced. Finally, in the $50 \%$ central-cue probability condition, there is no reason to select one location, and attention should not therefore be focused on one position. With these considerations in mind, it is possible to make several predictions regarding the relationship between central and peripheral cues.

If the central and peripheral cues influence different information-processing stages, this should be evident in, first, replication of the additivity between these variables (a replication of Experiments 1 and 2) and, second, no relationship between the informativeness of the central cue, where this may be perfect (i.e., $100 \%$ treatment), partial (i.e., $70 \%$ treatment), or nil (i.e., $50 \%$ treatment). By contrast, if central and peripheral cues involve the same information-processing stage, an interaction may be observed where the relative strength of the two cue types will determine which is more influential. The form of the interaction, then, depends on the nature of the relationship between the central and peripheral cues. The relationship could be inhibitory, in which case increasing central-cue informativeness would be negatively correlated with the magnitude of the peripheral-cue effect-that is, the difference between the invalid and valid peripheral-cue conditions.

Müller and Humphreys (1991) have described a similar form of interaction between the central and peripheral cues. Although their model assumes the existence of sep- 
arate mechanisms for spatial attention, the two orienting mechanisms interact in a mutually inhibitory way. When the voluntary mechanism is maximally engaged, it inhibits the reflexive mechanism, and the degree of inhibition is probably associated with the validity of the cue. The reflexive mechanism, on the other hand, is engaged, on an all-or-none basis, without reference to the validity of the central cue. In this sense, the two inhibitions have a different strength.

According to the position advanced by Müller and Humphreys (1991), then, the $100 \%$ probability of the centralcue condition should virtually eliminate peripheral cue effects. The opposite should be true of the $50 \%$ central condition, where the peripheral cue should have a strong effect. The $70 \%$ central condition could represent an intermediate situation if subjects focused attention on the centrally cued location on some percentage of trials.

The relationship between central-cue informativeness and the invalid and valid peripheral-cue conditions could be facilitatory, however. That is, the voluntary assignment of attention to one spatial location could increase the difference between the invalid and valid peripheral-cue treatments, as if the peripheral process were enhanced by the central process. A similar idea has been offered by Müller and Rabbitt (1989), who noted that "voluntary orienting, on the basis of the spatial cue (set validity), appears able to modify the effect of the reflexive mechanisms: to attenuate it when cue and flash are incompatible, and to enhance it when they are compatible" (pp. 327-328). It is possible, however, that they were referring to the impact of congruency on the addition and subtraction of their effects, and not to the limited-capacity attentional system.

\section{Method}

Subjects. Sixteen right-handed students ( 6 males and 10 females, 20-25 years of age) from the University of Parma were tested. They had normal or corrected-to-normal vision, were ignorant as to the purpose of the experiment, and were paid for their participation.

Apparatus and Procedure. A microcomputer (IBM PC/AT 386) was used for stimulus generation and response recording. The subject sat in front of the computer screen with his or her head resting on an adjustable head-and-chinrest and additionally restrained by the chair head-holder. The distance between the eyes and the screen was about $50 \mathrm{~cm}$. Eye position was monitored using an infrared oculometer, linearity range $\pm 15^{\circ}$ in horizontal direction and $\pm 10^{\circ}$ in vertical direction, resolution $1^{\prime}-5^{\prime}$ of arc (for more information, see Bach, Bouis, \& Fischer, 1983).

Responses were emitted by pressing one of two keys on the computer keyboard (left and right $<$ shift $>$ keys). The keys were in symmetrical positions in relation to the median sagittal axis and operated by the index finger of the two hands.

For the $70 \%$ central-validity condition, as in Experiments 1 and 2 , the cue was a plain arrow (tip formed by two lines) pointing right or left; in the $50 \%$ validity condition, the cue was a bidirected plain arrow, and in the $100 \%$ validity condition it was a bold arrow (the end of the arrow was a full triangle) pointing right or left. The cues were easily discriminable from each other. As in previous experiments, central and peripheral cues were presented in each trial and manipulated orthogonally. The peripheral cues consisted of an expansion of one of the two peripheral dots, which were uninformative of the side of stimulus presentation.

Table 4 shows the frequency of each type of trial in relation to the validity of the central cue, the side of presentation of the peripheral cue, and the position of the letters. Only the conjunction set was tested. The letters were inscribed in a $0.46^{\circ} \times 0.85^{\circ}$ matrix.

The experiment consisted of 16 blocks of 40 correct trials, run in two sessions, with some minutes of rest in between. The first experimental session was preceded by a series of eye-movement calibrations in the four main visual axes and a block of practice trials. Error responses were discarded and replaced.

As far as possible, the procedure and timing of events were similar to those in Experiment 1. Each trial started with a 1,000-msec presentation of the word <ready> on the screen; three dots were then shown with the peripheral dots always situated at $2.86^{\circ}$ to the right and left of the fixation point. After $500 \mathrm{msec}$, the fixation dot was replaced by the central cue $\left(0.69^{\circ} \times 0.46^{\circ}\right)$, which remained on view until the end of the trial. One thousand milliseconds later, one of the two peripheral dots expanded for $48 \mathrm{msec}$ in an area of $0.46^{\circ}$ $\times 0.46^{\circ}$. Following a delay of $102 \mathrm{msec}$, the letters were shown for $100 \mathrm{msec}$; between the letters there was a gap of $0.23^{\circ}$. In the other hemifield, at the corresponding area, a dotted pattern was presented.

The response ended the trial and was followed by a 1- to 2-sec feedback regarding speed and accuracy. RTs shorter than $150 \mathrm{msec}$ or longer than $1,200 \mathrm{msec}$ were considered errors, as were those trials in which an eye movement was detected or the response was made by a wrong keypress. The display remained on until a response occurred or until a 1,200-msec interval had elapsed since the letters first appeared.

Eye stability was controlled as follows: eye position was first measured at the moment of central-cue presentation and then compared with that starting from the offset of peripheral-cue presentation until the end of the trial was reached. If the difference exceeded $1^{\circ}$ in the horizontal direction, the trial was discarded and rerun. Eye movement occurred in less than $5 \%$ of the trials.

\section{Results}

ANOVAs were implemented on mean RT (correct responses) and accuracy (following arcsine normalization). The first ANOVA followed those described above for Experiments 1 and 2 for the $70 \%$ central-probability condition. A second analysis was conducted to examine the relationship between the probability of central-cue conditions $(100 \%, 70 \%$, or $50 \%)$ and the peripheral conditions (valid or invalid).

Reaction time: Analysis of the $70 \%$ probability of central-cue treatment. The first analysis involved the following factors: central cue (valid or invalid), peripheral cue (valid or invalid), hemifield (left or right), and response type (yes or no). As shown in Figure 3, the pattern of results was similar to those reported for Experi-

Table 4

Summary of Experiment 3 Design Specifying the Directional Value of Each Cue and the Frequency of Each Type of Trial

\begin{tabular}{|c|c|c|c|c|}
\hline \multirow{2}{*}{$\begin{array}{l}\text { Validity of } \\
\text { Central Cue }\end{array}$} & \multicolumn{2}{|c|}{ Type of Cue } & \multicolumn{2}{|c|}{ Position of Stimulus } \\
\hline & Central & Peripheral & Left & Right \\
\hline $70 \%$ & $\begin{array}{l}\text { Left } \\
\text { Left } \\
\text { Right } \\
\text { Right }\end{array}$ & $\begin{array}{l}\text { Left } \\
\text { Right } \\
\text { Left } \\
\text { Right }\end{array}$ & $\begin{array}{l}56 \\
56 \\
24 \\
24\end{array}$ & $\begin{array}{l}24 \\
24 \\
56 \\
56\end{array}$ \\
\hline $100 \%$ & $\begin{array}{l}\text { Left } \\
\text { Left } \\
\text { Right } \\
\text { Right }\end{array}$ & $\begin{array}{l}\text { Left } \\
\text { Right } \\
\text { Left } \\
\text { Right }\end{array}$ & $\begin{array}{r}56 \\
56 \\
0 \\
0\end{array}$ & $\begin{array}{r}0 \\
0 \\
56 \\
56\end{array}$ \\
\hline $50 \%$ & $\begin{array}{l}\text { Neutral } \\
\text { Neutral }\end{array}$ & $\begin{array}{l}\text { Left } \\
\text { Right }\end{array}$ & $\begin{array}{l}24 \\
24\end{array}$ & $\begin{array}{l}24 \\
24\end{array}$ \\
\hline Total & & & 320 & 320 \\
\hline
\end{tabular}


ments 1 and 2 . The main effects of central cue $[F(1,15)=$ $54.35, p<.01]$, peripheral cue $[F(1,15)=24.41, p<.01]$, and response type $[F(1,15)=44.58, p<.01]$, were significant. The interaction between central cue and peripheral cue was not significant $[F(1,15)=0.08, p>.25]$. The results are therefore consistent with the additivity hypothesis. The magnitudes of the main effects were 28 and $17 \mathrm{msec}$ for the central-and peripheral-cue conditions, respectively. The equivalent values for Experiment 1 were 25 and $21 \mathrm{msec}$, respectively.

No significant main effects of interactions were obtained in an ANOVA conducted on the accuracy data. Mean accuracy was $87.8 \%$ and $84.9 \%$ correct for the valid and invalid central-cue conditions, respectively, and $86.8 \%$ and $85.9 \%$ correct for the valid and invalid peripheral-cue conditions, respectively.

Reaction time: Comparison between the $50 \%, 70 \%$, and $100 \%$ probability of central-cue treatments. The second ANOVA was conducted to test the proposition that the magnitude of the difference between the valid and invalid peripheral-cue conditions was insensitive to variation in the probability of central cue. For this analysis, consideration was restricted to trials in which letters were actually presented on the side indicated by the central cue. The only trials therefore excluded from this analysis were the invalid trials from the $70 \%$ central invalid condition. The factors for this analysis were probability of central cue $(100 \%, 70 \%$, and $50 \%$ ), peripheral cue (valid or invalid), hemifield (left or right), and response type (yes or no). The critical interaction involving probability of central cue and peripheral cue was not significant $[F(2,30)=$ $1.15, p>.10$ ], although, as shown in Figure 4, the difference between the valid and the invalid peripheral-cue conditions increased systematically as a function of the probability of central cue.

Significant effects were also obtained for probability of the central cue $[F(2,30)=6.17, p<.01]$, peripheral cue $[F(1,15)=25.08, p<.01]$, hemifield $[F(1,15)=$ $17.62, p<.01]$, and response type $[F(1,15)=87.19, p<$ $.01]$. Trials were faster in the $100 \%$ condition than in the other conditions $(563,577$, and $572 \mathrm{msec}$ for the $100 \%$, $70 \%$, and $50 \%$ probability trials, respectively), showing that at least the $100 \%$ condition was treated differently from the other conditions. RT under the valid peripheralcue treatment was $19 \mathrm{msec}$ faster than RT under the invalid peripheral-cue treatment. RT for the right hemifield was $19 \mathrm{msec}$ faster than RT for the left hemifield, and yes responses were $61 \mathrm{msec}$ faster than no responses.

No significant main effects of interactions were obtained in an ANOVA conducted on the accuracy data. Overall, accuracy was $88.1 \%$.

\section{EXPERIMENT 4}

Experiment 4 was implemented to replicate and extend our claim that peripheral- and central-cue effects were additive under experimental conditions which also demonstrate that subjects use the informational value of
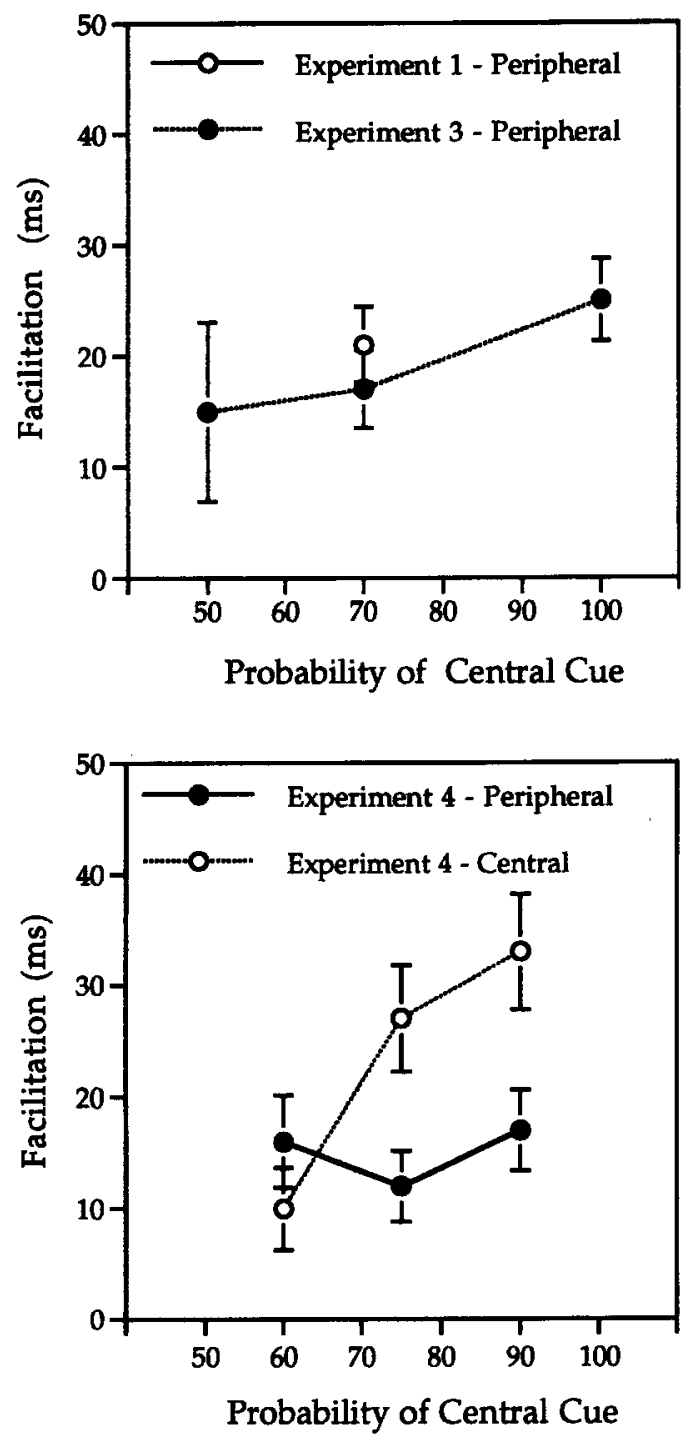

Figure 4. Peripheral facilitation (invalid-valid) as a function of central-cue probability for Experiments 1, 3, and 4. Central facilitation for Experiment 4 is also shown. Bars represent standard errors.

the central cue to direct voluntary attention. In Experiment 3 , the central-cue validity conditions were mixed, forcing subjects to change strategy from trial to trial according to the informativeness of the cue. The task was complex, and the instructions may have been difficult to follow. Experiment 4 was designed to overcome these problems. The same central cue was used for all probability treatments, and the subjects were provided with information about the informativeness of the central cue separately for each block of trials. This procedure allows for a direct measure of the benefit and cost as a function of central-cue validity. The additive hypothesis predicts that peripheral-cue effects will be observed in the presence of modulation of central-validity effects related to the informativeness of the central cue, but that they will be insensitive to these effects. 
Since the probability of central-cue levels used for Experiment $3,50 \%, 70 \%$, and $100 \%$, did not provide comparable data for all treatments, three new levels-60\%, $75 \%$, and $90 \%$ - were used for Experiment 4 . These levels should yield data for the valid and invalid treatments at each level of probability of central cue.

\section{Method}

Subjects. Twelve right-handed students ( 7 males and 5 females, 21-26 years of age) from the University of Parma participated in the experiment. They had normal or corrected-to-normal vision, were ignorant as to the purpose of the experiment, and were paid for their participation.

Apparatus and Procedure. The apparatus, stimuli, and procedure were similar to those of Experiment 3 . As before, central and peripheral cues were presented in each trial and manipulated orthogonally. The peripheral cues were formed by an expansion of one of the two peripheral dots, always uninformative of the side of stimulus presentation, but the area of the expansion was double that of Experiment 3.

Three central-cue conditions were used: $60 \%, 75 \%$, and $90 \%$ of probability to predict the side of the appearance of the letters. Each central-cue condition was in a different block of trials. To avoid sequence effects, presentation of the three block types was counterbalanced among subjects according to a Latin square design. The central cues always consisted of a plain arrow pointing right or left. The arrow was identical to the cue used in Experiment 3 for the $70 \%$ central-cue validity condition. The subjects were fully informed about the relevant cuing procedure and instructed to treat the arrow differently according to probability manipulation. Thus, the three cue-probability treatments differed only in regard to the instructions about cue probability and the percentage of occasions on which the cue predicted letter position.

Only the conjunction set was tested. The letters were presented until response. The experiment comprised 1,056 correct trials divided into blocks of about 40 correct trials, run in two sessions. There were 160 trials in the $60 \%$ probability condition, 256 trials in the $75 \%$ probability condition, and 640 trials in the $90 \%$ probability condition, giving a total of at least 8 trials for each combination of the variables.

Eye stability was controlled as in Experiment 3. Trials in which an eye movement occurred were less than $6 \%$.

\section{Results}

The correct mean response latencies and accuracies (following arcsine normalization) were entered into two ANOVAs with the following within-subjects factors: probability of central cue $(60 \%, 75 \%$, and $90 \%)$, central cue (valid and invalid conditions), peripheral cue (valid and invalid conditions), hemifield (left and right), and response type (yes and no).

Reaction time. The main effects of central cue $[F(1,11)$ $=78.57, p<.01]$ and peripheral cue $[F(1,11)=85.23$, $p<.01]$ were significant; the interaction between these two variables was not $[F(1,11)=0.66, p>.25]$. The interaction between probability of central cue and central cue was significant $[F(2,22)=6.11, p<.01]$, showing that subjects followed the instructions treating the arrow differently in the three probability situations. In particular (see Figure 4), there was an increase in the centralvalidity effects according to the strength of the central cue to predict the location of the letters. Although the central valid trials were always faster than the central in- valid trials, the difference was significant only in $75 \%$ and $90 \%$ probability trials ( 27 and $33 \mathrm{msec}$, respectively, both $p s<.01)$; in the $60 \%$ situation, the difference $(10 \mathrm{msec})$ did not reach significance $(.05<p<.10)$. On the contrary, the interactions involving probability of central cue and peripheral cue $[F(2,22)=0.47, p>.25]$ and probability of central cue, central cue, and peripheral cue $[F(2,22)=0.92, p>.25]$ were not significant. These results are in agreement with an additive model of the relationship between central cues and peripheral cues. As Figure 4 shows, only central-validity effects follow the informativeness of central cues; peripheral-validity effects do not.

Although mean accuracy was $91.8 \%, 93.7 \%$, and $92.8 \%$ in the $60 \%, 75 \%$, and $90 \%$ probability of central-cue conditions, respectively, there were no significant main effects or interactions in these data.

\section{GENERAL DISCUSSION}

\section{Summary of Results}

The results of the experiments were as follows. First, significant differences were obtained between the valid and invalid conditions for the peripheral cues in all four experiments. Second, significant differences were obtained between the valid and invalid conditions for the central cues in the three RT experiments, and, although it was not significant at $\alpha=.01$, the same pattern was observed in the threshold experiment. Third, the relationship between central and peripheral cues was additive in all four experiments. Fourth, there were no significant three-way interactions involving central cue, peripheral cue, and hemifield, response type, or letter set in the primary analyses involving the $70 \%$ probability of centralcue treatment. Fifth, the difference between the invalid and valid peripheral-cue treatments was statistically indifferent to the probability of central cue in Experiments 3 and 4 , a pattern which indicates that peripheral-cue facilitation was insensitive to the central-cue manipulation, although the results demonstrated that central-cue manipulation influenced performance directly.

An important aspect of the results not included in the above summary involves possible interactions between each of the cue types and the other variables. A review of the relevant analyses indicated that there were no consistent patterns, even with a less conservative alpha (.05). There were two significant interactions involving hemifield with the central-cue treatment in Experiment 1 and the peripheral-cue treatment in Experiment 2. There were no significant interactions involving response type (yes or no) and either of the cue types. Also, in the studies that included letter set, no significant interactions involving these variables and cue type were observed.

Task can also be thought of as a variable for this purpose, although the experiments were not designed to support a systematic comparison between the threshold and RT procedures. If anything, our finding that virtually the same patterns of main effects and interactions are ob- 
served for threshold and RT procedures suggests that they do not depend on the presence of an explicit speeded response in the task structure. Put in other words, the results suggest that the stages influenced by the central and peripheral cues do not depend on the presence and operation of an explicit response in the task demands.

\section{Validity Effects}

Significant differences were observed between the valid and invalid treatments for both central and peripheral cues. The result is of course congruent with the literature when the peripheral and central cues are considered separately provided that subjects focus voluntary attention under central-cue conditions and that peripheral cues capture attention. The situation is not clear when the two cues are contrasted during the same experimental trials. Our results are consistent with conditions in which the probability of the central cue is less than $100 \%$ (e.g., Koshino et al., 1992; Müller \& Rabbitt, 1989; Yantis \& Jonides, 1990). However, when the probability of the central cue was exactly $100 \%$, the results obtained have been different. Yantis and Jonides (1990), for example, observed central-cue effects in the absence of peripheral-cue effects, both inside and outside the focus of voluntary attention. Theeuwes (1991) also reported a central-cue effect, but he found either inhibition or no effect at all when the peripheral cue was inside the focus of voluntary attention, and no peripheral cue effect at all when the peripheral cue was outside the focus of voluntary attention. There are many differences among Experiment 3 (above) and the experiments of other researchers, and any one of these differences could account for the results. For example, the $50 \%, 70 \%$, and $100 \%$ probability of central-cue conditions were included in the same block of trials, contrary to the studies cited above. It is possible that voluntary control is fully effective only when it is tested under blocked conditions. Warner et al. (1990), for example, found that the symbolic status of peripheral cues could influence peripheral-cue effects, but only after extensive practice. Our results showed that the $100 \%$ central probability condition used here under mixed probability of central-cue conditions does not override peripheral-cue effects, even though it is associated with a reduction in RT relative to the $70 \%$ treatment.

There is another aspect of our study that should be emphasized. The peripheral cue used in Experiment 3 involved a small enlargement of a peripheral dot. This change was difficult to detect during the experimental trials. The peripheral cue used by Theeuwes (1991), for example, involved a line segment near the target and, because this was spatially adjacent to but distinct from the stimulus, it is possible that it subsequently competed with the target during stimulus analysis. The results of Theeuwes's second experiment are consistent with this hypothesis. When the peripheral cue was defined by offset of a line segment, the peripheral cue effect was abolished. In this case, then, there is no separate unit to compete for attention.

\section{Stages}

The results of the experiments are consistent with the proposition that peripheral and central cues exert their influence on performance through two independent stages of information processing. The main effect of cue validity was significant in virtually every case, and the interaction between cue type (central and peripheral) and cue validity (valid and invalid) was not significant in any case. The statistical results are therefore consistent with the model developed by Sternberg (1969) and reviewed by Miller (1988), under which the presence of an additive relationship between mean RT for two independent variables is consistent with the proposition that these variables influence separate information-processing stages.

Our conclusion about the relationship between the central and peripheral cues, however, should be treated with caution in one respect. In principle, additivity should be observed under multiple levels of each variable that could influence both mechanisms. Interstimulus interval is, of course, an obvious candidate. It is possible that a relationship that is additive when the central and peripheral cues are presented 1,140 and $140 \mathrm{msec}$ before the test stimulus, respectively, might collapse when other cue-totest stimulus onset asynchronies (SOAs) are used. It should be noted, however, that the range for which interaction might occur is circumscribed by the temporal patterns associated with central- and peripheral-cue effects. While peripheral-cue effects may be observed under SOA conditions as short as $100 \mathrm{msec}$ or less (Cheal \& Lyon, 1991; Müller \& Findlay, 1988; Müller \& Rabbitt, 1989), they are not observed at SOAs of greater than $200 \mathrm{msec}$. By comparison, central-cue effects may be observed at SOAs as short as $200 \mathrm{msec}$ (Cheal \&Lyon, 1991; Müller \& Findlay, 1988; Müller \& Rabbitt, 1989), but the outer limit is probably set by memory or concentration limits rather than attention. In our studies, we selected a SOA condition that reflected these figures, with the central cue leading the peripheral cue by approximately $1 \mathrm{sec}$. The specific SOA value used was selected to enable comparison with Briand and Klein (1987).

Figure 1 includes provisional revisions to Sanders's (1990) review. The figure should be considered in two steps. In the first, we have associated the peripheral and central cues with specific stages in Sanders's (1990) or Sternberg's (1969) model. Peripheral cuing is associated with early perceptual stages involving either preprocessing or feature extraction. As Experiments 1 and 2 did not reveal any interactions involving the critical variables and letter set, this classification must be treated as provisional.

The position in regard to central cuing is complex. It may be assumed that central cues, like other variables that involve voluntary or selective processes, influence a decision stage that is responsible for selecting a decision category to meet the specific and explicit demands of the task. This interpretation receives independent support from evidence that central-cue effects are selectively influenced by manipulation of memory load and instructions (Jonides, 1981). 
In summary, (1) the product of peripheral and central cuing influences distinct information-processing stages, (2) peripheral cues influence an early perceptual stage, and (3) central cues influence a late perceptual or, possibly, decision stage of information processing. The conclusion that both cues influence perceptual stages is based on the results of Experiment 2, where the relationship between the central- and peripheral-cue treatments was additive under threshold conditions that, it is assumed, excluded response factors from consideration.

\section{The Implicit Response Route}

Figure 1 has been refined to cope with evidence that peripheral and possibly central cues influence another response system, not just the manual response normally used to meet explicit task demands. In many respects, this problem can be compared to the more general issue concerning the distinction between explicit and implicit processes in other aspects of perception, learning, and memory. The explicit task demands require the subject to press one of two buttons to indicate whether or not a particular letter was present in the displays. The implicit demands for many tasks that require deployment and control of attention involve eye movements and, possibly, reorientation of effectors. People get ready to respond to new stimuli in spatially selective ways, where behavioral anticipation includes preparation of a motor program involving eye or manual movements. Then, if further action is required, the eye- or pointing-movement program can be executed.

The finding that peripheral and, possibly, central cues influence letter-identification thresholds under conditions in which the task demands do not include an explicit response requirement poses a major problem for selective attention models which implicate response planning, programming, or execution in their account of selectiveattention effects. The scale of the challenge is underlined by evidence that variables that must be associated with response stages - response hand for example - interact with peripheral cues. Consider the experiment described by Possamaï (1991), for example. Each trial included three stimulus events, a hand cue (e.g., an off-screen cue defining response hand), a peripheral stimulus cue (i.e., an outline square at $7^{\circ}$ to the left or right of fixation), and a target stimulus (i.e., a cross at $7^{\circ}$ to the left or right of fixation), and subjects were required to respond on a go/nogo basis to target presence/absence. Possamaï found peripheral facilitation when response hand was ipsilateral to the target, and inferred that the peripheral stimulus cue had its impact rather late, after the sensory coding of the stimulus. Now, when this argument is referred to a conventional stage analysis (the central column in Figure 1), it must be inferred that otherwise distant variables, such as response hand and peripheral cue, influence the same stage of the formal analysis as prescribed by the explicit task demands. But, if this is the case, stage analyses will be difficult to defend. The problem posed by the results of Experiment 2 addresses the same issue. In that experiment, performance is measured by threshold changes and there is no effective response requirement; yet peripheral- and, possibly, central-cue effects were obtained. One approach would be to claim that all cuing effects were perceptual, implying that accounts that emphasize response factors are incorrect in some unspecified way. We have adopted an alternative posture - that the observed interactions involving response factors reflect the integral and implicit role of response planning in perception.

Thus, the paradox associated with the assignment of "perceptual" variables to response stages of information processing can be resolved if provision is made for an additional and implicit information-processing route. RT models do not generally include provision for implicit responses. As developed by Sternberg (1969), Miller (1988), and Sanders (1990), for example, consideration is restricted to the explicit response route, the route that is represented in the right-hand column in Figure 1. However, Figure 1 includes a left-hand column for implicit responses as well. This column includes two stages for motor programming and motor adjustment that are equivalent to the final response stages in the explicit response route. But, while the latter are concerned with preparation and execution of the response to the explicit task demands, the former are concerned with responses to the implicit task demands, involving eye movement, body orientation, or pointing, for example. It may now be noted that the lefthand column involves processes that may be equivalent to the processes described by Rizzolatti, Riggio, and Sheliga (1994; see also Rizzolatti, Riggio, Dascola, \& Umiltà, 1987; Sheliga, Riggio, \& Rizzolatti, 1994; Umiltà, Riggio, Dascola, \& Rizzolatti, 1991). Presentation of a peripheral cue, for example, may trigger preparation of a motor program that may or may not subsequently be used to support a selective eye movement. According to the account advanced by Rizzolatti et al. (1994), selective spatial attention is based directly on preparatory processes of this type, hence the return arrow to the preprocessing stage. Thus, the process responsible for programming eye movement also changes the quality of the perceptual operation-here assumed to involve stimulus preprocessing - that will be applied to the spatial location for which an orientating movement has been prepared. Thus, the model outlined in Figure 1 includes an additional route to describe implicit response processes, where these involve a model predicated on the assumption that response preparation is the crucial process in selective attention. It should also be noted that although this revision was developed specifically to cater to eye movements, or planned eye movements, the additional route need not be confined to programs for eye movements. It could also be used to prepare other movements, ones involving the hands, or the head, for example.

However, although our model includes provision for implicit processes involving premotor planning, the contribution of this mechanism to selective attention does not necessarily transfer the balance of explanation to response stages that are involved in the preparation and production of the explicit response defined by task demands. Rather, it provides a more detailed articulation of the microprocesses that support selective processes dur- 
ing perception. Premotor planning is triggered directly by an early perceptual stage-involving preprocessing or detection, for example - and it, in turn, facilitates information processing efficiency in that perceptual stage.

The model depicted in Figure 1 includes an assumption that motor programming for the implicit response involves a distinct stage. This assumption is provisional. The motor-programming step could be treated as a component process in the preprocessing stage, for example. Another approach, consistent with our interpretation of Possamaï's (1991) study, involves an assumption that the explicit and implicit routes constitute an example of parallel processing, where the implicit route bypasses the explicit route and interacts with one or more stages subsequent to response selection. It should be conceded, however, that this interpretation challenges a basic assumption of the additive factor methodology - that the information-processing stages are serial and independent. Further research will be required to clarify this issue.

\section{REFERENCES}

BACH, M., BouIs, D., \& Fischer, B. (1983). An accurate and linear infrared oculometer. Journal of Neuroscience Methods, 9, 9-14.

Briand, K. A., \& KLein, R. M. (1987). Is Posner's "beam" the same as Treisman's "glue"?: On the relation between visual orienting and feature integration theory. Journal of Experimental Psychology: Human Perception \& Performance, 13, 228-241.

CAstiello, U., \& Umiltà, C. (1992). Splitting focal attention. Journal of Experimental Psychology: Human Perception \& Performance, 18, 837-848.

Cheal, M. L., \& LyON, D. R. (1991). Importance of precue location in directing attention. Acta Psychologica, 76, 201-211.

DownING, C. J. (1988). Expectancy and visual-spatial attention: Effects on perceptual quality. Journal of Experimental Psychology: Human Perception \& Performance, 14, 188-202.

ERIKSEN, C. W., \& Colegate, R. L. (1971). Selective attention and serial processing in briefly presented visual displays. Perception \& Psychophysics, 10, 321-326.

ERIKSEN, C. W., \& HofFMAN, J. I. (1972). Temporal and spatial characteristics of selective encoding from visual displays. Perception \& Psychophysics, 12, 201-204.

ERIKSEN, C. W., \& YEH, Y.-Y. (1985). Allocation of attention in the visual field. Journal of Experimental Psychology: Human Perception \& Performance, 11, 583-597.

Gawryszewski, L. DE G., Riggio, L., Rizzolatti, G., \& Umiltà, C. (1987). Movements of attention in the three spatial dimensions and the meaning of "neutral" cues. Neuropsychologia, 25, 19-29.

Hawkins, H. L., Hillyard, S. S., LuCK, S. J., Mouloua, M., DownING, C. J., \& Woodward, D. P. (1990). Visual attention modulates signal detectability. Journal of Experimental Psychology: Human Perception \& Performance, 16, 802-811.

HUMPHREYS, G. W., \& BRUCE, V. (1989). Visual cognition. Hillsdale, $\mathrm{NJ}$ : Erlbaum.

JONIDES, J. (1980). Towards a model of the mind's eye's movement. Canadian Journal of Psychology, 34, 103-112.

JONIDES, J. (1981). Voluntary versus automatic control over the mind's eye's movement. In J. B. Long \& A. D. Baddeley (Eds.), Attention and performance $I X$ (pp. 187-203). Hillsdale, NJ: Erlbaum.

Klein, R. M., Kingstone, A., \& Pontefract, A. (1992). Orienting of visual attention. In K. Raynor (Ed.), Eye movements and visual cognition: Scene perception and reading (pp. 46-65). New York: SpringerVerlag.

Koshino, H., Warner, C. B., \& Juola, J. F. (1992). Relative effectiveness of central, peripheral, and abrupt-onset cues in visual attention. Quarterly Journal of Experimental Psychology, 45A, 609-631.
Lieberman, H. R., \& Pentland, A. P. (1982). Microcomputer-based estimation of psychophysical thresholds: The best PEST. Behavior Research Methods \& Instrumentation, 14, 21-25.

MADDEN, D. J. (1992). Selective attention and visual search: Revision of an allocation model and application to age differences. Journal of Experimental Psychology: Human Perception \& Performance, 18, 821-836.

MAYLOR, E. A. (1985). Facilitatory and inhibitory components of orienting in visual space. In M. I. Posner \& O. S. M. Marin (Eds.), Attention and performance XI (pp. 189-204). Hillsdale, NJ: Erlbaum.

MAYLOR, E. A., \& HoCKEY, R. (1985). Inhibitory component of externally controlled covert orienting in visual space. Journal of Experimental Psychology: Human Perception \& Performance, 11, 777-787.

MiLlER, J. (1988). Discrete and continuous models of human information processing: Theoretical distinctions and empirical results. Acta Psychologica, 67, 191-257.

MÜLleR, H. J., \& Findlay, J. M. (1987). Sensitivity and criterion effects in the spatial cuing of visual attention. Perception \& Psychophysics, 42, 383-399.

Müller, H. J., \& FindLAY, J. M. (1988). The effects of visual attention on peripheral discrimination thresholds in single and multiple element displays. Acta Psychologica, 69, 129-155.

Müller, H. J., \& HuMPHREYS, G. W. (1991). Luminence-increment detection: Capacity limited or not? Journal of Experimental Psychology: Human Perception \& Performance, 17, 107-124.

MüLLER, H. J., \& RABBITT, P. M. A. (1989). Reflexive and voluntary orienting of visual attention: Time course of activation and resistance to interruption. Journal of Experimental Psychology: Human Perception \& Performance, 15, 315-330.

OLDFIELD, R. C. (1971). The assessment and analysis of handedness: The Edinburgh Inventory. Neuropsychologia, 9, 97-113.

POSNER, M. I. (1980). Orienting of attention. Quarterly Journal of Experimental Psychology, 32, 3-25.

PoSNER, M. I., \& COHEN, Y. (1984). Components of visual orienting. In H. Bouma \& D. G. Bouwhuis (Eds.), Attention and performance $X$ (pp. 55-66). Hillsdale, NJ: Erlbaum.

Posner, M. I., SNyder, C. R. R., \& Davidson, B. J. (1980). Attention and the detection of signals. Journal of Experimental Psychology: General, 109, 160-174.

Possamaï, C. A. (1986). Relationship between inhibition and facilitation following a visual cue. Acta Psychologica, 61, 243-258.

Possamaï, C. A. (1991). A responding hand effect in simple-RT precuing experiment: Evidence for a late locus of facilitation. Acta Psychologica, 77, 47-61.

Prinzmetal, W., Presti, D. E., \& Posner, M. I. (1986). Does attention affect visual feature integration? Journal of Experimental Psychology: Human Perception \& Performance, 12, 361-370.

Remington, R. W., Johnston, J. C., \& Yantis, S. (1992). Involuntary attentional capture by abrupt onsets. Perception \& Psychophysics, 51, 279-290.

Rizzolatti, G., Riggio, L., Dascola, I., \& Umiltà, C. (1987). Reorienting attention across the horizontal and vertical meridians: Evidence in favour of a premotor theory of attention. Neuropsychologia, 25, 31-40.

Rizzolatti, G., Riggio, L., \& Sheliga, B. M. (1994). Space and selective attention. In C. Umiltà \& M. Moscovitch (Eds.), Attention and performance $X V$ (pp. 231-265). Cambridge, MA: MIT Press.

SANDERS, A. F. (1990). Issues and trends in the debate on discrete vs. continuous processing of information. Acta Psychologica, 74, 123-167.

Sheliga, B. M., Riggio, L., \& Rizzolatti, G. (1994). Orienting of attention and eye movements. Experimental Brain Research, 98, 507-522.

STANDEN, P. (1990). Auditory and visual word recognition processes. Unpublished doctoral dissertation, University of Western Australia.

STERNBERG, S. (1969). The discovery of processing stages: Extensions of Donder's method. Acta Psychologica, 30, 276-315.

Tassinari, G., Aglioti, S., Chelazzi, L., Marzi, C. A., \& BerlucCHI, G. (1987). Distribution in the visual field of the costs of voluntarily allocated attention and of the inhibitory after-effects of covert orienting. Neuropsychologia, 25, 55-71.

THEEUWES, J. (1991). Exogenous and endogenous control of attention: 
The effect of visual onsets and offsets. Perception \& Psychophysics, 49, 83-90.

Treisman, A. (1985). Preattentive processing in vision. Computer Vision, Graphics, \& Image Processing, 31, 156-177.

Treisman, A., \& Gelade, G. (1980). A feature-integration theory of attention. Cognitive Psychology, 12, 97-136.

Treisman, A., \& SATo, S. (1990). Conjunction search revisited. Journal of Experimental Psychology: Human Perception \& Performance, 16, 453-478.

TREISMAN, A., \& SCHMIDT, H. (1982). Illusory conjunctions in the perception of objects. Cognitive Psychology, 14, 107-141.

UMiltì, C., \& Nicoletti, R. (1985). Attention and coding effects in S-R compatibility due to irrelevant spatial cues. In M. I. Posner \& O. S. M. Marin (Eds.), Attention and performance XI (pp. 457-471). Hillsdale, NJ: Erlbaum.
Umiltà, C., Riggio, L., Dascola, I., \& Rizzolatti, G. (1991). Differential effects of central and peripheral cues on the reorienting of spatial attention. European Journal of Cognitive Psychology, 3, 247267.

WARNER, C. B., JuOlA, J. F., \& Koshino, H. (1990). Voluntary allocation versus automatic capture of visual attention. Perception \& Psychophysics, 48, 243-251.

YANTIS, S., \& JoNIDES, J. (1990). Abrupt visual onsets and selective attention: Voluntary versus automatic allocation. Journal of Experimental Psychology: Human Perception \& Performance, 16, 121-134.

(Manuscript received August 2, 1993; revision accepted for publication August 16, 1996.) 